\title{
A solitary hyperfunctioning thyroid nodule harboring thyroid carcinoma: review of the literature
}

\author{
Sasan Mirfakhraee ${ }^{1}$, Dana Mathews², Lan Peng 3 , Stacey Woodruff ${ }^{4}$ and Jeffrey M Zigman ${ }^{1 *}$
}

\begin{abstract}
Hyperfunctioning nodules of the thyroid are thought to only rarely harbor thyroid cancer, and thus are infrequently biopsied. Here, we present the case of a patient with a hyperfunctioning thyroid nodule harboring thyroid carcinoma and, using MEDLINE literature searches, set out to determine the prevalence of and characteristics of malignant "hot" nodules as a group. Historical, biochemical and radiologic characteristics of the case subjects and their nodules were compared to those in cases of benign hyperfunctioning nodules. A literature review of surgical patients with solitary hyperfunctioning thyroid nodules managed by thyroid resection revealed an estimated 3.1\% prevalence of malignancy. A separate literature search uncovered 76 cases of reported malignant hot thyroid nodules, besides the present case. Of these, $78 \%$ were female and mean age at time of diagnosis was 47 years. Mean nodule size was $4.13 \pm 1.68 \mathrm{~cm}$. Laboratory assessment revealed $\mathrm{T}_{3}$ elevation in $76.5 \%, \mathrm{~T}_{4}$ elevation in $51.9 \%$, and subclinical hyperthyroidism in $13 \%$ of patients. Histological diagnosis was papillary thyroid carcinoma (PTC) in $57.1 \%$, follicular thyroid carcinoma (FTC) in 36.4\%, and Hurthle cell carcinoma in $7.8 \%$ of patients. Thus, hot thyroid nodules harbor a low but non-trivial rate of malignancy. Compared to individuals with benign hyperfunctioning thyroid nodules, those with malignant hyperfunctioning nodules are younger and more predominantly female. Also, FTC and Hurthle cell carcinoma are found more frequently in hot nodules than in general. We were unable to find any specific characteristics that could be used to distinguish between malignant and benign hot nodules.
\end{abstract}

Keywords: Hot nodule, Thyroid cancer, Malignancy

\section{Introduction}

Thyroid nodules are frequently-encountered entities in clinical practice, occurring with a prevalence of $4 \%$ by palpation [1], $33 \%$ to $68 \%$ by ultrasound examination $[2,3]$, and $50 \%$ on autopsy series [4]. While approximately $95 \%$ of thyroid nodules are benign, certain historical, laboratory, and sonographic features raise the suspicion for malignancy [5]. As the initial step for evaluation of a thyroid nodule is measurement of serum thyroid stimulating hormone (TSH) [6,7], it is not uncommon for patients with a solitary thyroid nodule to be diagnosed with hyperthyroidism. In this setting, the thyroid nodule may represent a solitary hyperfunctioning

\footnotetext{
* Correspondence: jeffrey.zigman@utsouthwestern.edu

'Department of Internal Medicine, Division of Endocrinology and Metabolism, The University of Texas Southwestern Medical Center, Dallas, Texas 75390, USA

Full list of author information is available at the end of the article
}

thyroid nodule in an otherwise normal thyroid gland or it may represent a hyperfunctioning or nonfunctioning nodule occurring within a toxic multinodular goiter, within a Graves' disease or destructive thyroiditis milieu, or in an individual with a less common cause of thyrotoxicosis [8]. Thyroid scintigraphy employs radioiodine $\left({ }^{123} \mathrm{I},{ }^{131} \mathrm{I}\right)$ or technetium-99m- $\left({ }^{99 \mathrm{~m}} \mathrm{Tc}\right)$ pertechnetate in order to differentiate these diagnostic possibilities. The distinction is important, because hyperfunctioning nodules - also referred to as "autonomous," "autonomouslyfunctioning," or "hot" nodules - are thought to only rarely harbor malignancy, such that fine needle aspiration (FNA) is not traditionally indicated in this circumstance [6]. Per the 2009 revised American Thyroid Association Management Guidelines for Patients with Thyroid Nodules and Differentiated Thyroid Cancer, "Since hyperfunctioning nodules rarely harbor malignancy, if one is

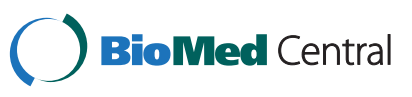


found that corresponds to the nodule in question, no cytologic evaluation is necessary" [6].

Here, we present the case of a woman with subclinical hyperthyroidism due to a hyperfunctioning thyroid nodule who was diagnosed with minimally-invasive follicular carcinoma after surgical resection. We also include our findings from a formal literature review on this topic, in which the historical, laboratory, and radiological features of similarly-documented cases were scrutinized to determine if there are features that differentiate hyperfunctioning thyroid carcinomas from solitary toxic adenomas. The results of a separate literature review aimed at estimating the prevalence of thyroid cancer within hot thyroid nodules are also presented. Our goal is to call attention to the fact that hyperfunctioning thyroid carcinomas are welldescribed in the literature (and also likely underreported), challenging the commonly-held notion that the hot thyroid nodule is very unlikely to be cancerous.

\section{Materials and methods}

A MEDLINE literature search of English-language studies published between 1950 and January 2012 with the terms, "thyroid cancer, hyperthyroidism, surgery," "thyroid cancer, hyperfunctioning nodule, surgery," and "thyroid cancer, hot nodule, surgery," was performed to determine the reported prevalence of thyroid carcinoma in patients undergoing resection of solitary hyperfunctioning thyroid nodules. Another literature search, using the terms, "hyperfunctioning thyroid carcinoma," "toxic adenoma, thyroid carcinoma," and "hot nodule, thyroid carcinoma" was performed using MEDLINE and by reviewing the citations of relevant articles in order to collect data on reported cases of patients with a solitary thyroid nodule found to harbor thyroid carcinoma. Case series were included provided that at least some demographic and clinical details of individual subjects were described. A third MEDLINE literature search, using the terms "hot nodule," "hyperfunctioning thyroid nodule," and "autonomous thyroid nodules," was performed to establish the demographic characteristics and nodule sizes of subjects with solitary hyperfunctioning thyroid nodules. In this third group, the available studies analyzed the group of hyperfunctioning nodules en masse and did not make a distinction between hyperfunctioning nodules that were benign and those that may have been malignant. Therefore, while the aggregate data from these studies most likely involved predominantly benign cases, a small number of malignant cases also may have been included. For this reason, we will subsequently refer to this group as "predominantly-benign" hyperfunctioning (or hot) nodules.

Weighted averages of data were calculated by assigning a "weight" to each study (based on the number of subjects in the study divided by the total number of subjects for all studies), multiplying each weight by the subject mean in the corresponding study, and then taking the sum of these products.

\section{Results}

\section{Patient case}

A 29-year-old teacher was referred to her local endocrinologist to evaluate a palpable left thyroid nodule. While she did not report thyrotoxic symptoms at that time, she was found to have a suppressed TSH. Thyroid scintigraphy revealed a hyperfunctioning left-sided thyroid nodule, and ultrasonography revealed it to be $2.4 \mathrm{~cm}$ in greatest dimension, isoechoic, and in the left lower lobe. A follow-up ultrasound one year later revealed an essentially unchanged nodule measuring $2.5 \mathrm{~cm}$.

The patient presented to our institution the next year, at which time she endorsed tremors, anxiety, insomnia, and oligomenorrhea. She denied local compressive symptoms, history of radiation exposure, or family history of thyroid malignancy. Her only medication was a daily multivitamin. Her weight was $106 \mathrm{~kg}$ with a height of 1.7 $\mathrm{m}$, blood pressure of $117 / 73 \mathrm{mmHg}$, and heart rate of 79 beats per minute. A $1.5 \mathrm{~cm}$, firm, slightly tender nodule was palpated in the left lower lobe of the thyroid; it moved with swallowing. There was no evidence of cervical lymphadenopathy or thyroid bruit. The patient had a slight, fine tremor of the hands with normal biceps deeptendon reflexes bilaterally.

Thyroid function tests revealed a suppressed TSH (0.005 mcIU/mL, normal $0.4-4.5 \mathrm{mcIU} / \mathrm{mL})$ but normal free T4 (1.1 ng/dL, normal $0.9-1.8 \mathrm{ng} / \mathrm{dL})$ and free T3 (3.5 pg/mL, normal $2.3-4.2 \mathrm{pg} / \mathrm{mL}$ ). Thyroid peroxidase antibody testing was negative $(0.4 \mathrm{IU} / \mathrm{mL}$, normal $<9$ $\mathrm{IU} / \mathrm{mL})$, as were thyroglobulin antibody $(<20 \mathrm{IU} / \mathrm{mL})$ and thyroid stimulating immunoglobulin $(<1.0)$ testing. Thyroid ultrasound revealed a $2.6 \times 2.7 \times 2.6 \mathrm{~cm}$ predominantly solid, isoechoic left lower lobe nodule with internal hypervascularity within a slightly enlarged but otherwise normal-appearing thyroid gland (Figure $1 \mathrm{~A}$ and $\mathrm{B}) .{ }^{123} \mathrm{I}$ thyroid scintigraphy revealed a left lower lobe hyperfunctioning nodule with 24-hour uptake of 27\% (Figure 1C).

After discussing with the patient the benefits and risks of radioiodine ablation versus surgical resection (left hemithyroidectomy), she elected for the latter, citing the reduced risk of permanent hypothyroidism as her main deciding factor. Histologic evaluation of the surgical specimen revealed a solitary, circumscribed, and wellencapsulated tumor measuring $2.5 \times 2.5 \times 2.2 \mathrm{~cm}$ without lymphovascular invasion or extra-thyroidal extension (Figure 1D). A focus was identified where the tumor penetrated and budded through the well-defined fibrous capsule, giving the diagnosis of minimally-invasive follicular thyroid carcinoma (Figure 1E) in the setting of background lymphocytic thyroiditis. Given the pathologic 


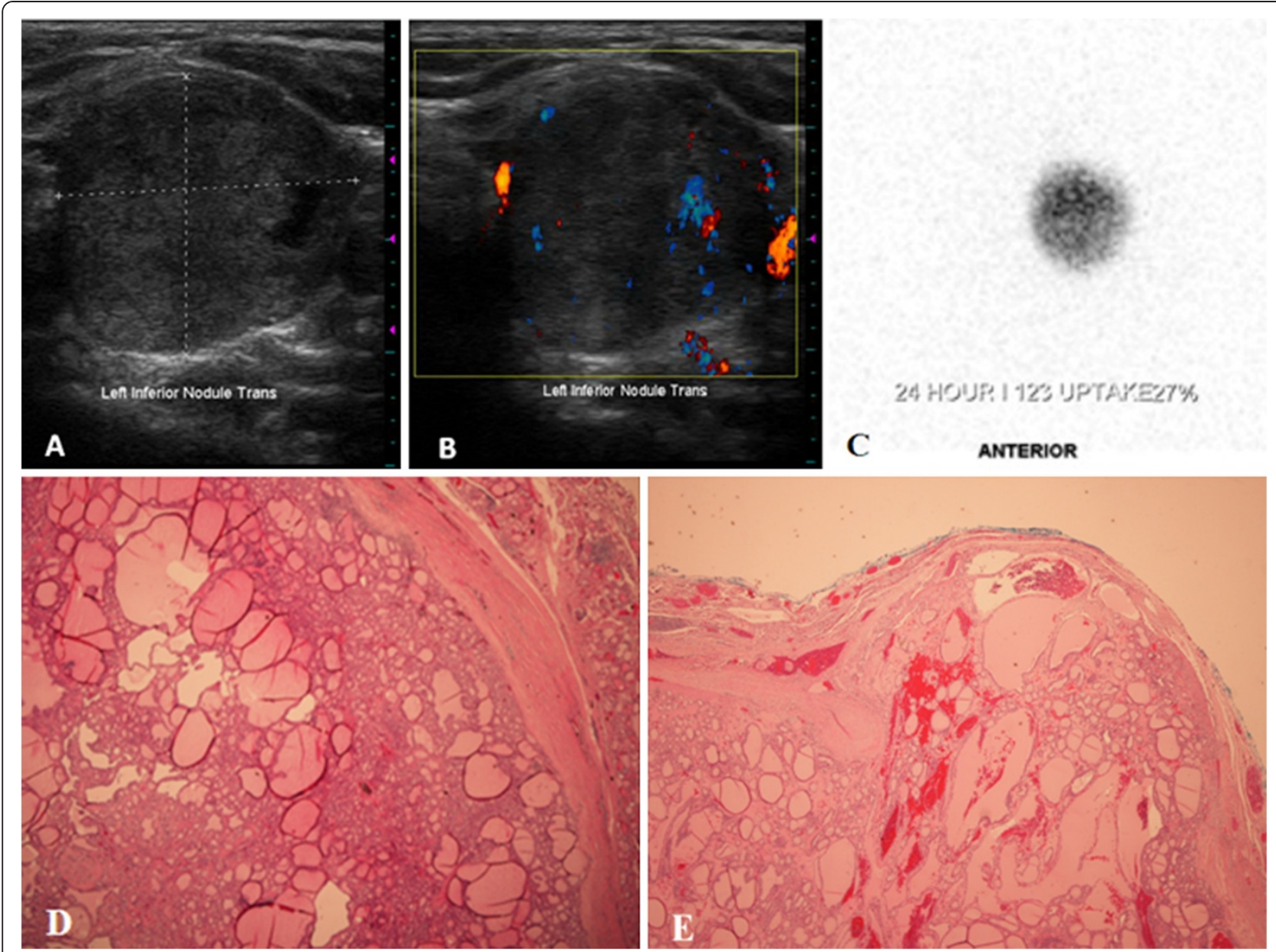

Figure 1 Imaging and histologic features of the hot nodule present in the case report subject. (A) Ultrasonography of the left thyroid lobe, demonstrating a $2.7 \mathrm{~cm}$, predominantly solid, and isoechoic nodule. (B) Color Doppler evaluation reveals blood flow within the rim of the nodule and intraparenchymally. (C) ${ }^{123}$ thyroid scintigram depicts a round left-sided focus of iodine uptake with suppression in the remainder of the gland, consistent with an autonomously-functioning thyroid nodule. (D) Histological evaluation reveals that the lesion is solitary, circumscribed and encapsulated. The follicular proliferation is surrounded by a rather thick fibrous capsule. The lesion demonstrates a predominant follicular pattern of growth without papillary cytologic features (hematoxylin-eosin stain; original magnification $\times 4$ ). (E) A focal area is identified where the tumor invades through and into the fibrous capsule (hematoxylin-eosin stain; original magnification $\times 2$ ).

diagnosis, lobectomy was felt to be sufficient, and neither completion thyroidectomy nor radioactive iodine ablation was pursued in this patient. Molecular testing was performed on the surgical specimen for BRAF and KRAS mutations, both of which were negative. At her 6-month follow up appointment, the patient was symptomatically and biochemically euthyroid on low-dose levothyroxine replacement and showed no evidence of cancer recurrence.

\section{Estimated prevalence of malignancy within hot thyroid nodules}

In order to place the current case report in context, we have attempted to establish the prevalence of malignancy within hot thyroid nodules. This involved a literature search for surgical case series of solitary hyperfunctioning nodules managed by thyroid resection. A total of
14 relevant case series were uncovered. The earliest we found was from 1967 and included 79 hot nodules that underwent surgical resection; none of those cases were found to harbor thyroid carcinoma. Among the 14 case series, carcinoma rates of intranodular carcinoma ranging from $0-12.5 \%$ were noted, with a weighted total of $3.1 \%$ (Table 1). Of note, some of those case series also included cases in which thyroid carcinoma occurred outside of the hot nodule; however for the purposes of our review, such extranodular cases were not included in our estimated prevalence determination.

\section{Search for distinctive features of malignant hot thyroid nodules}

We next sought to establish historical and/or clinical features that may help to differentiate malignant, 
Table 1 Intranodular thyroid carcinoma prevalence in patients undergoing resection of solitary, hyperfunctioning thyroid nodules

\begin{tabular}{|c|c|c|c|}
\hline Author & $\begin{array}{c}\text { Cases of } \\
\text { hyperfunctioning } \\
\text { nodule }(\mathrm{n})\end{array}$ & $\begin{array}{l}\text { Cases of thyroid } \\
\text { carcinoma within } \\
\text { the hyperfunctioning } \\
\text { nodule }(\mathrm{n})\end{array}$ & $\begin{array}{c}\text { Thyroid } \\
\text { carcinoma } \\
\text { prevalence } \\
(\%)\end{array}$ \\
\hline Giles [9] & 176 & 4 & 2.3 \\
\hline Cakir [10] & 63 & 4 & 6.3 \\
\hline Cappelli [11] & 207 & 3 & 1.4 \\
\hline Foppiani [12] & 16 & 2 & 12.5 \\
\hline Sahin [13] & 77 & 2 & 2.6 \\
\hline Gabriele [14] & 120 & 2 & 1.7 \\
\hline Harach [15] & 73 & 6 & 8.2 \\
\hline Vaiana [16] & 153 & 3 & 2 \\
\hline Zanella [17] & 41 & 3 & 7.3 \\
\hline Terzioglu [18] & 25 & 2 & 8 \\
\hline Pacini [19] & 40 & 1 & 2.5 \\
\hline Smith [20] & 30 & 2 & 6.7 \\
\hline $\begin{array}{l}\text { Hamburger } \\
{[21]}\end{array}$ & 24 & 1 & 4.2 \\
\hline Horst [22] & 79 & 0 & 0 \\
\hline Total & 1124 & 35 & 3.1 \\
\hline
\end{tabular}

Only cases of intra-nodular malignancy are included. Studies are arranged in order of ascending date of publication.

$\mathbf{n}=$ number of case.

hyperfunctioning thyroid nodules from benign, toxic adenomas, especially since hyperfunctioning thyroid carcinoma is generally an ex post facto diagnosis. Using the search criteria listed in the Methods Section, we discovered 76 cases (in addition to the current case) of malignant hot thyroid nodules, in which autonomy was highly suggested by both biochemical parameters of hyperthyroidism and scintigraphic evidence of increased radioiodine or labeled technetium uptake. All possibly-relevant features of the 77 cases were extracted and are displayed in Table 2, and a further analysis of these features appears in Table 3. An additional 27 cases with scintigraphic evidence suggestive of nodular autonomy but with normal thyroid function tests, absent thyroid function testing, or uninterpretable laboratory results (e.g., patients already on levothyroxine therapy at time of laboratory collection) were also found in the literature search (Table 4); these cases were excluded from subsequent analyses since autonomy seemed less certain.

\section{Demographic characteristics}

We compared age at diagnosis and female: male ratio of individuals with malignant, hyperfunctioning thyroid nodules to those with benign, toxic adenomas. Data on the latter group was compiled from a separate literature search which identified several surgical case series of predominantly-benign hot nodules, as described in the Methods section (Table 5). Subjects with malignant hot nodules were younger at the time of diagnosis than those listed in multiple case series of predominantly-benign hot nodules [47.0 vs. 57.6 years, respectively (Tables 3 and 5)]. Additionally, a greater percentage of subjects with malignant hot nodule were female as compared to those with predominantly-benign hot nodules [3.53:1 vs. $1.65: 1$ female to male ratio, respectively (Tables 3 and 5)].

\section{Size}

The sizes of the malignant hot nodules (Table 2) and predominantly-benign hot thyroid nodules are compared in Figure 2A. Of note, the clinical use of ultrasonography began in the late 1960s, so that the sizes listed in the early case reports of malignant hot nodule were estimated by palpation. We include tumors that were noted to encompass the entirety of the hot nodule as well as microcarcinomas embedded within a larger hot nodule. The actual size of the tumor within the thyroid nodule determined by pathological review was occasionally provided in the literature and is listed in Tables 2 and 4 . The mean nodule size among the subjects with thyroid carcinoma was $4.13 \pm 1.68 \mathrm{~cm}$, which closely approximates the mean size of the predominantly-benign hyperfunctioning nodules.

\section{Biochemical profile}

The majority of subjects with a malignant hot nodule demonstrated elevation of triiodothyronine (76.5\%), whereas closer to half of the subjects had elevation of thyroxine (51.9\%) (Table 3). Note that these percentages represent the number of subjects with an elevated thyroid hormone level (total and/or free value, depending on the particular study) divided by the total number of subjects with a hyperfunctioning thyroid nodule. Far fewer subjects had subclinical hyperthyroidism (13\%) (Table 3). In comparison, with the exception of 3 studies, similar data on the biochemical profiles of subjects with benign, toxic adenoma in the collected case series were only sparsely included. In a study of 35 subjects with toxic adenoma, Hamburger found 16 with elevations in both $\mathrm{T}_{3}$ and $\mathrm{T}_{4}$ levels, 16 with elevations of only $\mathrm{T}_{3}, 3$ with isolated $\mathrm{T}_{4}$ excess, and 7 with subclinical hyperthyroidism [77]. In a study of 63 patients with solitary toxic adenoma, Langer found that mean free $\mathrm{T}_{3}$ level was significantly higher $(8.8 \pm 3.5 \mathrm{pg} / \mathrm{mL}$, normal range $4-6.8$ $\mathrm{pg} / \mathrm{mL})$ than mean free $\mathrm{T}_{4}$ level $(16.9 \pm 6.6 \mathrm{pg} / \mathrm{mL}$, normal range $7-17.5 \mathrm{pg} / \mathrm{mL}$ ) [78]. Blum evaluated 35 patients with solitary autonomous thyroid nodule and found that $65 \%$ had elevated $\mathrm{T}_{3}$ levels, $54 \%$ had elevated $\mathrm{T}_{4}$ levels, and $31 \%$ were biochemically euthyroid [72]. Thus, hypersecretion of $\mathrm{T}_{3}$ appears to be a common factor among both benign and malignant hyperfunctioning 
Table 2 Reported cases of biochemically-hyperthyroid patients with a reported hyperfunctioning nodule discovered to harbor thyroid carcinoma on pathological review

\begin{tabular}{|c|c|c|c|c|c|c|c|c|c|c|c|c|c|c|}
\hline$\#$ & Age & Sex & $\begin{array}{l}\text { Tumor growth } \\
(\mathbf{c m})\end{array}$ & High risk history $^{a}$ & Suspicious $\mathrm{U} / \mathrm{S}^{b}$ & $\begin{array}{c}\begin{array}{c}\text { Nodule size } \\
(\mathrm{cm})\end{array} \\
\end{array}$ & $\begin{array}{c}T^{\text {Tumor size }}{ }^{d} \\
(\mathrm{~cm})\end{array}$ & $\mathrm{TFTs}^{e}$ & $\begin{array}{c}\text { Toxic } \\
\text { sx? }\end{array}$ & $\begin{array}{c}\text { Compression } \\
\text { sx? }\end{array}$ & $\begin{array}{l}\text { Scan } \\
\text { type }\end{array}$ & FNA & $\begin{array}{c}\text { Surgical } \\
\text { path }\end{array}$ & $\begin{array}{c}\text { Reference } \\
\text { (1st author) }\end{array}$ \\
\hline 1 & 29 & $\mathrm{~F}$ & & - & IV & 2.7 & 2.5 & SHT & + & - & 123 & & FTC & Current case \\
\hline 2 & 43 & $\mathrm{~F}$ & & - & - & 6.5 & 5 & $\mathrm{fT}_{3}, \mathrm{fT}_{4}$ & + & - & Tc & & Hurthle & Karanchi [23] \\
\hline 3 & 13 & $\mathrm{~F}$ & & - & IV & 3.5 & 5 & $\Pi_{3}$ & - & - & 123 & & Hurthle & Yalla [24] \\
\hline 4 & 63 & M & & - & - & 4 & & $\mathrm{fT}_{4}$ & - & - & 123 & $\begin{array}{l}\text { Suspicion } \\
\text { of FVPTC }\end{array}$ & FVPTC & Bommireddipalli [25] \\
\hline 5 & 68 & $\mathrm{~F}$ & & - & $\mathrm{HE}$ & 5.3 & & $\mathrm{fT}_{3}$ & + & + & $T c_{1}^{123}$ & & FTC & Giovanella [26] \\
\hline 6 & 11 & $\mathrm{~F}$ & & - & - & 3.5 & & $\Pi_{3}$ & - & - & 123 & Nonspecific & PTC & Tfayli [27] \\
\hline 7 & 47 & $\mathrm{~F}$ & & - & - & 2.6 & 3 & $\Pi_{3,}, \mathrm{fT}_{4}$ & + & - & ${ }^{131}$ | & PTC & FVPTC & Azevedo [28] \\
\hline 8 & 36 & M & $1.4 \rightarrow 1.8$ in $11 \mathrm{mo}$ & - & $H E, I V$ & 1.8 & 1.5 & SHT & + & - & ${ }^{131}$ & PTC & PTC & Uludag [29] \\
\hline 9 & 62 & $\mathrm{~F}$ & & & - & & 2 & $\mathrm{fT}_{3}, \mathrm{fT}_{4}$ & + & - & Tc & PTC & PTC & Nishida [30] \\
\hline 10 & 32 & M & & - & - & 4.3 & & $\mathrm{fT}_{3}, \mathrm{fT}_{4}$ & - & - & Tc & Benign & FVPTC & Kim [31] \\
\hline 11 & 64 & $\mathrm{~F}$ & & - & & 6 & & $\Pi_{3}$ & + & - & ${ }^{131} \mid$ & & FTC & Niepomniszcze [32] \\
\hline 12 & 57 & $\mathrm{~F}$ & & & - & 6 & & $\operatorname{High}^{f}$ & + & - & Tc & Nondiagnostic & FTC & Bitterman [33] \\
\hline 13 & 59 & $\mathrm{~F}$ & & & - & 5 & & High & + & & Tc & & FTC & Bitterman [33] \\
\hline 14 & 59 & $\mathrm{~F}$ & & - & $\mathrm{HE}, \mathrm{PD}, \mathrm{Cal}$ & 1.5 & 1.5 & $\mathrm{fT}_{3}, \mathrm{fT}_{4}$ & + & + & $T c_{1}^{123}$ & PTC & PTC & Majima [34] \\
\hline 15 & NA & $\mathrm{F}$ & & & & 5 & 5 & $\mathrm{fT}_{3}, \mathrm{fT}_{4}$ & + & + & Tc & & PTC & Gozu [35] \\
\hline 16 & 67 & $\mathrm{~F}$ & & & & 2.5 & 3 & $\mathrm{fT}_{4}$ & + & - & Tc & Benign & Hurthle & Wong [36] \\
\hline 17 & 39 & $\mathrm{~F}$ & Subjective $\uparrow$ & & - & 2 & & $\mathrm{Nl} \rightarrow \mathrm{SHT}$ & - & $-\rightarrow+$ & 123 & & PTC & Yaturu [37] \\
\hline 18 & 36 & M & $2 x$ size in $5 y$ rs & & - & 2.8 & 2.3 & SHT & - & & & $\begin{array}{l}\text { Follicular } \\
\text { neoplasm }\end{array}$ & FVPTC & Logani [38] \\
\hline 19 & 11 & F & & - & - & & 4 & $\Pi_{3}, \mathrm{fT}_{4}$ & + & - & $T c_{1}^{131} \mid$ & & PTC & Mircescu [39] \\
\hline 20 & 49 & $\mathrm{~F}$ & & & & 4 & 3.5 & $\mathrm{fT}_{4}$ & + & - & ${ }^{123} \mid$ & & FTC & Camacho [40] \\
\hline 21 & 47 & M & & & & 3.5 & 3.5 & $\mathrm{fT}_{3}$ & + & & 123 & Suspicious & PTC & Bourasseau [41] \\
\hline 22 & 36 & M & & & & 2.5 & 2.5 & SHT & & & 123 & Nondiagnostic & FTC & Bourasseau [41] \\
\hline 23 & 56 & M & & & & 5.5 & 5.5 & $\mathrm{fT}_{4}$ & + & & 123 & & FTC & Bourasseau [41] \\
\hline 24 & 39 & F & & & & 1 & 1 & $\mathrm{fT}_{3}, \mathrm{fT}_{4}$ & + & & 123 & Suspicious & PTC & Bourasseau [41] \\
\hline 25 & 33 & $\mathrm{~F}$ & & & & 3 & 3 & SHT & & & 123 & Nondiagnostic & PTC & Bourasseau [41] \\
\hline 26 & 42 & $\mathrm{~F}$ & $\begin{array}{c}4.5 \rightarrow 7.4 \\
\text { (no interval given) }\end{array}$ & - & - & 7.4 & & SHT & - & - & 123 & Benign & Hurthle & Russo [42] \\
\hline 27 & 17 & $\mathrm{~F}$ & & - & HE & 2.1 & 2.1 & $\Pi_{3}$ & - & - & $T c_{1}{ }^{123}$ & & PTC & Cirillo [43] \\
\hline 28 & 60 & $\mathrm{~F}$ & & & - & 5 & 6 & $\Pi_{3}$ & + & - & ${ }^{131} \mid$ & & Insular & Russo [44] \\
\hline 29 & 16 & $\mathrm{~F}$ & & - & & 2 & & $\Pi_{4}$ & + & - & 123 & Colloid & Hurthle & Siddiqui [45] \\
\hline
\end{tabular}


Table 2 Reported cases of biochemically-hyperthyroid patients with a reported hyperfunctioning nodule discovered to harbor thyroid carcinoma on pathological review (Continued)

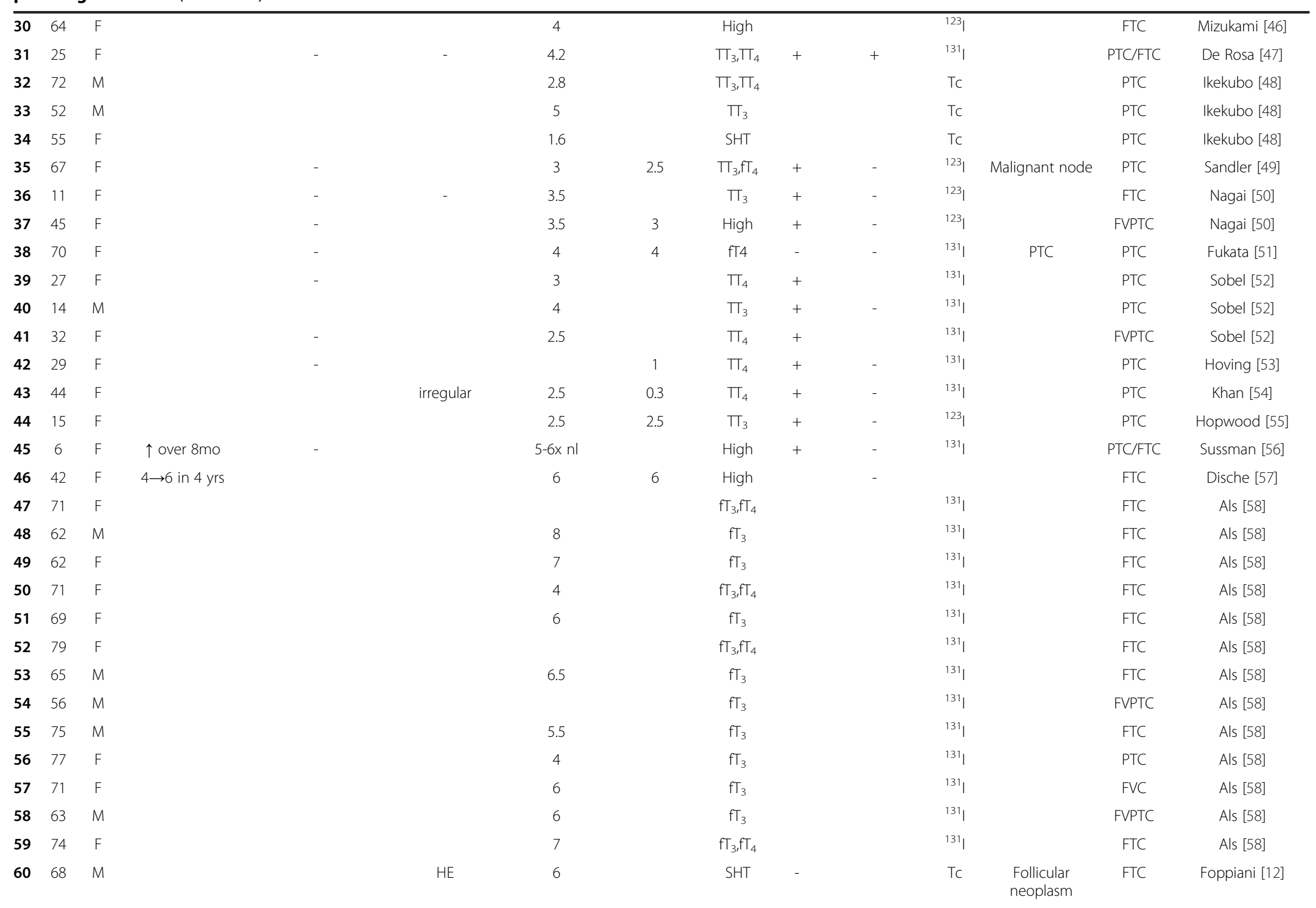


Table 2 Reported cases of biochemically-hyperthyroid patients with a reported hyperfunctioning nodule discovered to harbor thyroid carcinoma on pathological review (Continued)

\begin{tabular}{|c|c|c|c|c|c|c|c|c|c|c|c|c|c|}
\hline 61 & 38 & $\mathrm{~F}$ & & & HE & 2.7 & & SHT & & $\mathrm{TC}$ & $\begin{array}{l}\text { Hyperplastic } \\
\text { goiter }\end{array}$ & FTC & Foppiani [12] \\
\hline 62 & 35 & $\mathrm{~F}$ & & - & & & $>1 \mathrm{~cm}$ & High & + & ${ }^{131} \mid$ & PTC & PTC & Sahin [13] \\
\hline 63 & 65 & $\mathrm{~F}$ & & - & & & $>1 \mathrm{~cm}$ & High & + & ${ }^{131} \mid$ & PTC & PTC & Sahin [13] \\
\hline 64 & 19 & $\mathrm{~F}$ & & & & 5 & & $\Pi_{4}, \Pi_{3}$ & & & & PTC & Lin [59] \\
\hline 65 & 38 & $\mathrm{~F}$ & & - & - & & 0.3 & $\Pi_{3}$ & + & ${ }^{131} \mid$ & no malignancy & PTC & Taneri [60] \\
\hline 66 & 44 & F & & - & Cal & & 1 & $\pi_{3}$ & + & 131 & no malignancy & PTC & Taneri [60] \\
\hline 67 & 56 & $\mathrm{~F}$ & & - & & & 0.8 & High & & ${ }^{131} \mid$ & & FTC & Gabriele [14] \\
\hline 68 & 21 & $\mathrm{~F}$ & & - & IV & & 1.6 & High & & 131 & & FTC & Gabriele [14] \\
\hline 69 & 57 & $\mathrm{~F}$ & & - & - & & 0.7 & High & & & & PTC & Vaiana [16] \\
\hline 70 & 58 & $\mathrm{~F}$ & & - & - & & 3 & High & & & & PTC & Vaiana [16] \\
\hline 71 & 51 & F & & - & - & & 0.6 & High & & & & PTC & Vaiana [16] \\
\hline 72 & 17 & $\mathrm{~F}$ & & - & & & 1 & High & + & & & PTC & Pacini [19] \\
\hline 73 & 65 & F & Subjective $\uparrow$ & & & 5 & & High & + & & & FTC & Terzioglu [18] \\
\hline 74 & 42 & $\mathrm{~F}$ & & & & & & High & + & & & PTC & Terzioglu [18] \\
\hline 75 & 35 & $\mathrm{~F}$ & & - & & 2.2 & 0.5 & High & & & & PTC & Zanella [17] \\
\hline 76 & 70 & $\mathrm{~F}$ & & - & & 4.1 & 0.5 & High & & & & PTC & Zanella [17] \\
\hline 77 & 35 & M & & - & & 5.4 & 0.5 & High & & & & Hurthle & Zanella [17] \\
\hline
\end{tabular}

Abbreviations: + = yes; - = no; Cal = microcalcifications; FNA = fine needle aspiration; FTC = follicular thyroid carcinoma; FVPTC = follicular variant of papillary thyroid carcinoma; $\mathrm{HE}=$ hypoechoic; ${ }^{123}$ I = Iodine-123; ${ }^{131} \mathrm{I}$ $=$ lodine-131; IV = internal vascularity; $\mathrm{LT}_{4}=$ levothyroxine; $\mathrm{NA}=$ not available; $\mathrm{nl}=$ normal; $\mathrm{PD}=$ poorly demarcated; $\mathrm{PTC}=$ papillary thyroid carcinoma; $\mathrm{sX}=$ symptoms; $\mathrm{SHT}=$ subclinical hyperthyroidism; $\mathrm{fT} 3=$ free triiodothyronine; $\mathrm{fT}_{4}=$ free thyroxine; $\Pi_{3}=$ total triiodothyronine; $\Pi_{4}=$ total thyroxine; ${ }^{99 \mathrm{~m}} \mathrm{Tc}=$ technetium-99m-pertechnetate; $\mathrm{TFTs}=$ thyroid function testing; $\mathrm{U} / \mathrm{S}=\mathrm{ultrasound} ; \mathrm{XRT}=$ external beam radiotherapy. ${ }^{a}$ High-risk history: ionizing radiation exposure as child/adolescent, prior personal history of thyroid cancer, and family history of thyroid cancer in one or more 1st-degree relatives; as per Cooper et al. [6]. ${ }^{b}$ Suspicious ultrasound: hypoechoic, microcalcifications, increased nodular vascularity, poorly demarcated; as per Cooper et al. [6].

${ }^{c}$ Nodule size: The largest diameter of the thyroid nodule measured by ultrasonography, or if ultrasound not available, then by palpation.

${ }^{d}$ Tumor size: The largest diameter of the thyroid nodule measured grossly after surgical resection.

e TFTs: Indicates which thyroid hormone values (total T3, total T4, free $\mathrm{T}_{3}$, and/or free $\mathrm{T}_{4}$ ) were elevated at time of presentation, as opposed to SHT or euthyroidism. Of note, for many of these cases, no mention of one or more of these four standard thyroid hormone values was included.

${ }^{f}$ High: Indicates that the patient was biochemically hyperthyroid, though specific thyroid hormone levels were not given. 
Table 3 Demographic and clinical characteristics of the reported cases of hyperthyroid patients with hyperfunctioning thyroid carcinoma from the literature and the current case $(\mathbf{n}=77)$

\begin{tabular}{l} 
Characteristic \\
\hline Age \\
Mean-yr \\
Distribution - no. (\%) \\
$<15 \mathrm{yr}$ \\
$15-30 \mathrm{yr}$ \\
$31-45 \mathrm{yr}$ \\
$46-60 \mathrm{yr}$ \\
$>60 \mathrm{yr}$ \\
Sex- no. (\%) \\
Female \\
Male
\end{tabular}

$47.0 \pm 19.8^{*}$

High risk features

Historical - no. (\%)

$6(7.9 \%)$

$10(13.2 \%)$

$20(26.3 \%)$

$15(19.7 \%)$

$25(32.9 \%)$

$60(77.9 \%)$

$17(22.1 \%)$

Ultrasonographical - no. (\%)

Thyroid nodule size (via ultrasound or palpation) $(\mathrm{cm})$

Thyroid carcinoma size on pathological review

Mean $(\mathrm{cm})$

No. (\%) with size $<1 \mathrm{~cm}$

Biochemical hyperthyroidism

$\mathrm{T}_{3}$ elevated - no. (\%)

$\mathrm{T}_{4}$ elevated - no. (\%)

Subclinical hyperthyroidism - no. (\%)

Thyrotoxic symptoms - no. (\%)

Compression symptoms - no. (\%)

Thyroid scintigraphy

Technetium-99m-pertechnetate - no. (\%)

lodine-123 or -131 - no. (\%)

Fine-needle aspiration

Benign - no. (\%)

Malignant (or suspicious findings) - no. (\%)

Follicular neoplasm - no. (\%)

Nondiagnostic sample - no. (\%)

FNA accordant with final pathological diagnosis - no. (\%)

FNA not accordant with final pathological diagnosis - no. (\%)

Type of thyroid carcinoma on surgical pathological review

Follicular thyroid carcinoma

$28(36.4 \%)$

Papillary thyroid carcinoma

$44(57.1 \%)$

Follicular variant of papillary thyroid carcinoma

$8(18.2 \%)$

Hurthle cell carcinoma

$6(7.8 \%)$

Insular cell carcinoma

$1(1.3 \%)$

* Mean \pm standard deviation.

no. $=$ Number of subjects with this characteristic, $\%=$ percentage of subjects with this characteristic. thyroid nodules. Of interest, a greater prevalence of frank biochemical hyperthyroidism can be seen in patients with malignant hyperfunctioning thyroid nodules who have larger nodules (Figure 2B).

\section{Historical and sonographic features}

We next turned our attention to high-risk historical features and suspicious sonographic features to determine if these were present in cases of malignant hot nodule (Table 3). These features are described in Cooper et al. [6] and are listed in the Tables 2 and 3 legend. None of the 77 subjects with malignant hot nodules was noted to have high-risk historical features. Eleven subjects (36.7\%) had suspicious features on ultrasonography, which is likely an underestimate, as some of the more newlyrecognized high-risk sonographic features (e.g., taller than wide on transverse view) were not utilized in the earlier published reports. It was difficult to assess cases for nodule growth as a risk factor for malignancy, since the vast majority of the subjects were referred to surgery for immediate resection. However in seven cases, nodules were noted to grow over time. While an increase in nodule size is an indication for biopsy, the specificity of this finding for malignancy is limited, as $9-89 \%$ of benign nodules have been shown to grow over time depending on which definition for significant growth is used $[21,73,77,79]$.

\section{Histologic subtype}

The majority of malignant hot nodules were proven to be PTC $(57.1 \%)$, and of these, $18.2 \%$ were the follicular variant of PTC (FVPTC). Follicular thyroid carcinoma (as seen in our patient) comprised $36.4 \%$ of cases, while Hurthle cell carcinoma was found in $7.8 \%$ of samples. By comparison, in the U.S. National Cancer Data Base (1985-1995), which includes histologic information on all thyroid nodules as a group, the prevalence of PTC was approximately $85 \%$, FTC was $10 \%$, and Hurthle cell carcinoma was nearly $3 \%$ [80]. Thus, there does seem to be a higher prevalence of both FTC and Hurthle cell carcinoma in the hyperfunctioning thyroid carcinoma cases.

Of note, within the 77 identified cases of malignant hot nodule, only 23 subjects received FNA of their thyroid nodule prior to resection. FNA enabled a preoperative diagnosis of thyroid carcinoma in $43.5 \%$ of cases. However, $30.4 \%$ of subjects undergoing FNA were erroneously characterized as having benign lesions, and $17.4 \%$ of samples were nondiagnostic. Of the subjects with false negative biopsies, surgical pathology eventually revealed PTC in 3 cases (one of these was FVPTC), FTC in 1 case, and Hurthle cell carcinoma in 3 cases. Tumor size was given in only 3 instances of false negative biopsies. Two of these were of subcentimetric PTC, 
Table 4 Additional cases with scintigraphic evidence suggestive of an autonomous thyroid nodule without documented hyperthyroidism (or already on levothyroxine replacement therapy) discovered to harbor thyroid carcinoma on pathologic review

\begin{tabular}{|c|c|c|c|c|c|c|c|c|c|c|c|c|c|c|}
\hline$\#$ & Age & Sex & $\begin{array}{c}\text { Tumor } \\
\text { growth }(\mathrm{cm})\end{array}$ & $\begin{array}{l}\text { High risk } \\
\text { history }^{a}\end{array}$ & $\begin{array}{l}\text { Suspicious } \\
U / S^{b}\end{array}$ & $\begin{array}{l}\text { Nodule size } \\
(\mathrm{cm})\end{array}$ & $\begin{array}{c}\text { Tumor size }^{d} \\
(\mathrm{~cm})\end{array}$ & $\mathrm{TFTs}^{e}$ & Toxic sx? & Compression sx? & $\begin{array}{l}\text { Scan } \\
\text { type }\end{array}$ & FNA & $\begin{array}{l}\text { Surgical } \\
\text { path }\end{array}$ & $\begin{array}{l}\text { Reference } \\
\text { (1st author) }\end{array}$ \\
\hline 1 & 51 & $\mathrm{~F}$ & $2.7 \rightarrow 5.3$ in $2 y r s$ & - & - & 5.3 & 5 & on $\mathrm{LT}_{4}$ & - & + & $T c_{1}^{131} \mid$ & $\begin{array}{l}\text { Follicular } \\
\text { neoplasm }\end{array}$ & $\begin{array}{l}\text { Poor diff } \\
\text { cancer }\end{array}$ & Low [61] \\
\hline 2 & 44 & $\mathrm{~F}$ & & - & & 3.5 & 3.7 & $\mathrm{nl}$ & - & $-\rightarrow+$ & Tc & Benign & FTC & Schneider [62] \\
\hline 3 & 47 & M & & & & 1.4 & 1 & $\mathrm{nl}$ & - & & 123 & & PTC & Bourasseau [41] \\
\hline 4 & 34 & $\mathrm{~F}$ & & & & 1 & 1 & $\mathrm{nl}$ & - & & ${ }^{123} \mid$ & "Cancer" & PTC & Bourasseau [41] \\
\hline 5 & 37 & $\mathrm{~F}$ & & & & 1.5 & 1.5 & $\mathrm{nl}$ & - & & 123 & Nondiagnostic & FTC & Bourasseau [41] \\
\hline 6 & 39 & M & & & & 3 & & $\mathrm{nl}$ & & & 123 & & FVPTC & Mizukami [46] \\
\hline 7 & 69 & $\mathrm{~F}$ & & Prior PTC & & 4 & 3.3 & on $\mathrm{LT}_{4}$ & + & - & ${ }^{131}$ & & Hurthle & Caplan [63] \\
\hline 8 & 39 & M & & & $\mathrm{HE}, \mathrm{PD}, \mathrm{Cal}$ & & 1.5 & $\mathrm{nl}$ & - & - & 123 & & PTC & Michigishi [64] \\
\hline 9 & 65 & $\mathrm{~F}$ & & & & 4.3 & & $\mathrm{nl}$ & & & Tc & & PTC & Ikekubo [48] \\
\hline 10 & 37 & $\mathrm{~F}$ & & & & 2.5 & & $\mathrm{nl}$ & & & Tc & & PTC & Ikekubo [48] \\
\hline 11 & 39 & $\mathrm{~F}$ & & & & 3.5 & & $\mathrm{nl}$ & & & Tc & & PTC & Ikekubo [48] \\
\hline 12 & 38 & $\mathrm{~F}$ & & & & 4.5 & & $\mathrm{nl}$ & & & Tc & & PTC & Ikekubo [48] \\
\hline 13 & 35 & $\mathrm{~F}$ & & - & & 1 & 0.4 & $\mathrm{nl}$ & - & - & 123 & & PTC & Rubenfeld [65] \\
\hline 14 & 51 & M & & XRT & & "large" & & on $\mathrm{LT}_{4}$ & - & & 123 & & FTC & Nagai [50] \\
\hline 15 & 19 & $\mathrm{~F}$ & $4 \times 2 \rightarrow 4 \times 3$ in $1 \mathrm{yr}$ & & & 4 & 4 & $\mathrm{nl}$ & - & - & ${ }^{131}$ & & PTC/FTC & Abdel-Razzak [66] \\
\hline 16 & 15 & $\mathrm{~F}$ & & - & & & & $\mathrm{nl}$ & - & - & Tc & & PTC & Scott [67] \\
\hline 17 & 27 & $\mathrm{~F}$ & & & - & 4 & 2.3 & $\mathrm{nl}$ & + & - & ${ }^{131}$ & & PTC & Fujimoto [68] \\
\hline 18 & 21 & $\mathrm{~F}$ & & & & 3 & 1 & NA & + & + & ${ }^{131}$ & & PTC & Becker [69] \\
\hline 19 & 23 & $\mathrm{~F}$ & & & & 1.5 & 1 & NA & - & - & ${ }^{131}$ & & PTC & Becker [69] \\
\hline 20 & 28 & M & & & & 4.5 & 0.5 & NA & + & - & ${ }^{131}$ | & & PTC & Molnar [70] \\
\hline 21 & 54 & M & & & & 8.5 & & NA & & & ${ }^{131} \mid$ & & FTC & Als [58] \\
\hline 22 & 62 & $\mathrm{~F}$ & & & & & & NA & & & ${ }^{131} \mid$ & & PTC & Als [58] \\
\hline 23 & 61 & M & & & & & & NA & & & ${ }^{131}$ & & FTC & Als [58] \\
\hline 24 & 50 & M & & & & 10 & & NA & & & ${ }^{131}$ & & FTC & Als [58] \\
\hline 25 & 65 & $\mathrm{~F}$ & & & & 5 & & NA & & & ${ }^{131}$ | & & FTC & Als [58] \\
\hline 26 & 55 & $\mathrm{~F}$ & & & & 5.5 & & NA & & & 131 & & FTC & Als [58] \\
\hline
\end{tabular}


Table 4 Additional cases with scintigraphic evidence suggestive of an autonomous thyroid nodule without documented hyperthyroidism (or already on levothyroxine replacement therapy) discovered to harbor thyroid carcinoma on pathologic review (Continued)

\begin{tabular}{cccccccccccc}
\hline $\mathbf{2 7}$ & 66 & $\mathrm{~F}$ & & Cal & & $\mathrm{nl}$ & - & + & TC, \\
$\mathbf{7 7}$ & 35 & $\mathrm{M}$ & - & & 5.4 & 0.5 & High $^{f}$ & Colloid goiter & PTC & Bitterman [33] \\
\hline
\end{tabular}

Abbreviations: $+=$ yes; $-=$ no; $\mathrm{Cal}=$ microcalcifications; FNA $=$ fine needle aspiration; $\mathrm{FTC}=$ follicular thyroid carcinoma; FVPTC $=$ follicular variant of papillary thyroid carcinoma; $\mathrm{HE}=$ hypoechoic; ${ }^{123} \mathrm{I}=$ lodine-123; ${ }^{131} \mathrm{I}$ $=$ lodine-131; IV $=$ internal vascularity; $\mathrm{LT}_{4}=$ levothyroxine; $\mathrm{NA}=$ not available; $\mathrm{nl}=$ normal; $\mathrm{PD}=$ poorly demarcated; $\mathrm{PTC}=$ papillary thyroid carcinoma; $\mathrm{sX}=$ symptoms; $\mathrm{SHT}=$ subclinical hyperthyroidism; $\mathrm{fT} \mathrm{T}_{3}=$ free
triiodothyronine; $\mathrm{fT}_{4}=$ free thyroxine; $\Pi_{3}=$ total triiodothyronine; $\Pi_{4}=$ total thyroxine; $99 \mathrm{~m} \mathrm{TC}=$ technetium- $99 \mathrm{~m}$-pertechnetate; $\mathrm{TFTs}=$ thyroid function testing; $\mathrm{U} / \mathrm{S}=\mathrm{ultrasound} ; \mathrm{XRT}=$ external beam radiotherapy. ${ }^{a}$ High-risk history: ionizing radiation exposure as child/adolescent, prior personal history of thyroid cancer, and family history of thyroid cancer in one or more 1st-degree relatives; as per Cooper et al. [6]. ${ }^{b}$ Suspicious ultrasound: hypoechoic, microcalcifications, increased nodular vascularity, poorly demarcated; as per Cooper et al. [6].

c Nodule size: The largest diameter of the thyroid nodule measured by ultrasonography, or if ultrasound not available, then by palpation.

${ }^{d}$ Tumor size: The largest diameter of the thyroid nodule measured grossly after surgical resection.

e TFTs: Indicates which thyroid hormone values (total T3, total T4, free $\mathrm{T}_{3}$, and/or free $\mathrm{T}_{4}$ ) were elevated at time of presentation, as opposed to $\mathrm{SHT}$ or euthyroidism. Of note, for many of these cases, no mention of one

or more of these four standard thyroid hormone values was included.

or more of these four standard thyroid hormone values was included.
$f$ High: Indicates that the patient was biochemically hyperthyroid, though specific thyroid hormone levels were not given. 
Table 5 Demographic characteristics of patients with solitary hyperfunctioning thyroid nodules

\begin{tabular}{lcc}
\hline Author & Mean age (years) & Female: male (n) \\
\hline Bransom [71] & $49.7 \pm 13.4$ & $33: 35$ \\
Blum [72] & $50.9 \pm 17.5$ & $31: 4$ \\
Burch [73] & $45.8 \pm 16.8$ & $42: 13$ \\
Cappelli [11] & 61.8 & $445: 381$ \\
Giles [9] & $48.5 \pm 11.4$ & $147: 29$ \\
Hamburger [21] & $60 \pm 15$ & $19: 5$ \\
Iwata [74] & $48.8 \pm 15.4$ & $44: 0$ \\
Landgarten [75] & 49.3 & $106: 11$ \\
Linos [76] & 40 & $53: 9$ \\
Sahin [13] & $58.3 \pm 13.7$ & $50: 27$ \\
Smith [20] & 34.8 & $24: 6$ \\
Vaiana [16] & 61.8 & $340: 290$ \\
Weighted average & $\mathbf{5 7 . 6}$ & $\mathbf{1 . 6 5 : 1}$ \\
\hline
\end{tabular}

and it is presumed that small size was likely a chief factor leading to misdiagnosis. However, the other was a 3 cm nodule comprised wholly of Hurthle cell carcinoma.

\section{Discussion}

To our knowledge, the current data set is the largest and most detailed to date of patients with malignant hot thyroid nodule. In that regard, this study complements the informative and excellent 2012 review by PazaitouPanayiotou and colleagues examining the association of thyroid carcinoma with a broader spectrum of hyperthyroid states, including Graves' disease and toxic multinodular goiter in addition to hyperfunctioning thyroid nodule [81]. Of note, although it was not the focus of the Pazaitou-Panayiotou et al. review to perform a detailed analysis of the historical and clinical features of malignant hot nodule cases, as we did here, PazaitouPanayiotou and colleagues did include an evaluation of thyroid carcinoma prevalence. The reported percentages of thyroid carcinoma in their collected case series of patients with hot nodules, which overlapped but did not mirror exactly those case series evaluated here and which also included some cases of thyroid carcinoma occurring in extranodular thyroid tissue, ranged between $2.5-12.0 \%$. This corresponds to a weighted average of $6.9 \%$ and thus is similar to the $3.1 \%$ prevalence estimated here. In its discussion of thyroid carcinoma in patients with hyperfunctioning nodules, the Pazaitou-Panayiotou et al. review also included several other important commentaries. For instance, they draw attention to two studies suggestive of a higher prevalence of thyroid carcinoma in hot nodules occurring in children $[82,83]$, though the latter study included children from an iodine-deficient region. In addition, they discuss several reports in which activating mutations of the TSH receptor gene were identified within malignant hot nodules [81].

There are several noteworthy discussion points and implications to the findings presented here. The first relates to the prevalence of malignancy within solitary hot nodules. As mentioned, in the available surgical case series that addressed this topic, a varied prevalence was noted, ranging from $0-12.5 \%$, with a weighted average of $3.1 \%$. Only a minority of the patients who underwent surgery in those series did so because of concerning findings from FNA, and as such, we do not believe that the data set is biased towards cases in which a postoperative diagnosis of malignancy was expected. We realize that this collection of case series includes only a small fraction of the total number of solitary hot nodules that have occurred, and also that the vast majority of malignant hot nodules likely have gone unreported. Furthermore, it is likely that malignancy goes undiagnosed in many cases of hot nodules treated with radioiodine, especially those harboring microcarcinomas. Despite these limitations, we do believe there are sufficient numbers of cases included among the collected 14 surgical case series of subjects to cite the $3.1 \%$ figure as being a fair and representative estimate of malignancy prevalence amongst solitary hyperfunctioning nodules. While this $3.1 \%$ prevalence figure is low - and in fact is lower than the estimated $5-15 \%$ prevalence of malignancy among all thyroid nodules [6] - it is not trivial. Thus, the possibility of malignancy within a hot nodule must not be overlooked by a managing clinician, particularly if management other than surgical resection is chosen.

Another important discussion point regards the limitations resulting from the retrospective nature of this analysis, including incomplete data and differing methodology used in many of the collected case reports and case series of malignant hot nodule. While some of the primary sources were meticulous in their case descriptions, others included less-thorough descriptions. For example, tumor size was frequently not reported, and thus it was unclear if a PTC tumor was simply an incidental microcarcinoma embedded within a larger hot nodule or a large, follicular variant of PTC comprising the entirety of the nodule. While we have included some cases in which the malignancy was a microcarcinoma and thus of uncertain clinical significance [84], these cases are clearly in the minority (only 8 of the 77 reported tumors were less than one centimeter in size). Other factors affecting the data set are the evolution of technology and our understanding of risk factors for thyroid carcinoma. For instance, thyroid ultrasound began clinical use in the late 1960s, and prior to this, nodule size was estimated by palpation. Earlier reports would not have commented on some of the suspicious 


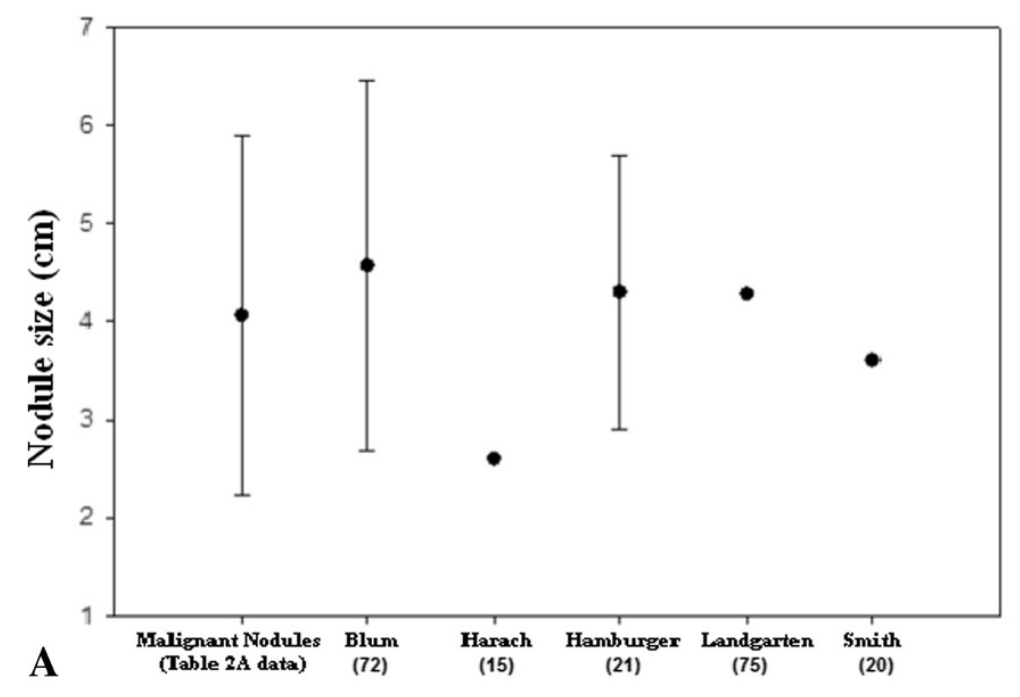

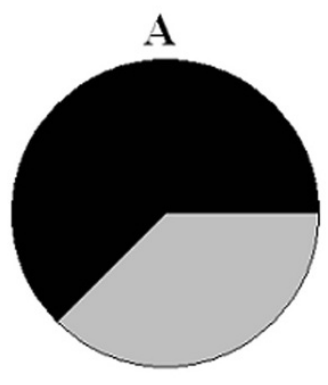

B
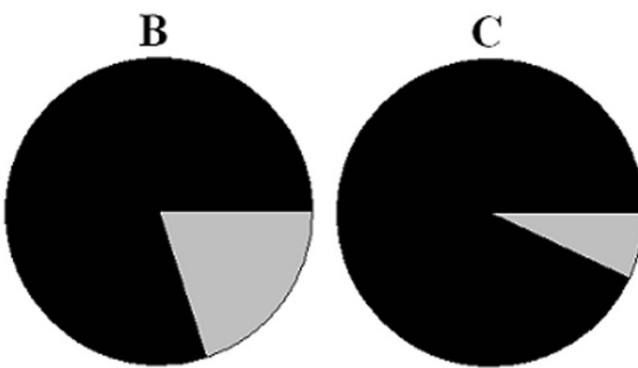

Hyperthyroidism

Subclinical hyperthyroidism

Figure 2 Size and biochemical assessment of hyperfunctioning thyroid nodules. (A) The mean greatest dimension of the malignant hot thyroid nodules from our case series is compared with that from five published surgical cases series of solitary, hyperfunctioning thyroid nodules. (B) The proportion of subjects with scintigraphically-determined hyperfunctioning thyroid carcinoma (Tables 2) who have frank biochemical hyperthyroidism vs. subclinical hyperthyroidism, based on varying nodule size. Subjects are characterized as having nodules $<2.5 \mathrm{~cm}(\mathbf{A}), 2.5-4.5$ $\mathrm{cm}(\mathbf{B})$, and $>4.5 \mathrm{~cm}(\mathbf{C})$ in diameter.

sonographic features recognized today. Additionally, the radioimmunoassays used to measure TSH have undergone many generations of refinement over the past several decades, and thus the presence and degree of hyperthyroidism may have been underestimated in earlier studies.

Also worth discussing are the differential prevalences of thyroid carcinoma histologic subtype found in hot nodules as compared to nodules as a group. As mentioned, there was a much higher prevalence of both FTC and Hurthle cell carcinoma in hot nodules $(36.4 \%$ and $7.8 \%$, respectively) as compared to in all nodules (10\% and nearly $3 \%$, respectively). Additionally, a substantial percentage of the PTC cases found in hot nodules were the follicular variant. This may have bearing on the current algorithm for evaluation of patients with thyroid nodules as recommended in the 2009 thyroid nodule and thyroid carcinoma management guidelines [6]. In particular, for those nodules found by biopsy to have follicular neoplasm by histology, the guidelines recommend consideration be made for performing an ${ }^{123}$ I thyroid scan, if not already done, especially if the serum TSH is in the low-normal range; if the nodule is found to be hyperfunctioning, it can then be followed [6]. However, the high prevalence of both FTC and FVPTC reported for malignant hot nodules suggests that a biopsy diagnosis of follicular neoplasm within a hot nodule may not be as reassuring as previously thought. Also of interest, in a 2009 study, Sundaraiya and colleagues reported a case of metastatic FTC occurring in the setting of thyrotoxicosis, in which high-grade extrathyroidal uptake of technetium-99m-pertechnetate was observed; in a literature search, they found 74 other cases of thyrotoxicosis resulting from well-differentiated thyroid cancer metastatic lesions, most of which demonstrated histologic evidence of FTC [85]. 
As a final point of discussion, since none of the historical, biochemical or radiologic characteristics that were assessed seems to predict malignancy in the collected cases of hot nodules, one might ask if there is any utility in biopsying hot nodules. Such would be a shift from the current thyroid nodule management guidelines which views an increased nodular radiotracer uptake pattern as a reassuring characteristic from a cancer perspective [6]. Given the estimated 3.1\% prevalence of malignancy within hot nodules, and also taking into account the difficulty in predicting whether a particular hot nodule is malignant, we recommend that hot nodules that are not treated surgically (as is an option to manage the hyperthyroidism) be considered for biopsy if high-risk historical and/or suspicious sonographic features are present or if these nodules grow over time, just as is currently recommended for nodules that are not hyperfunctioning [6]. This should also include previously hyperfunctioning nodules treated with radioiodine, although the known occurrence of dystrophic calcification and cystic degeneration as sequelae of radioiodine ablation should be taken into account when assessing their sonographic features [86]. Future prospective studies could help determine if biopsying at the time of diagnosis all hot nodules with suspicious sonographic characteristics and/or associated high-risk historical features versus biopsying hot nodules only if the initial sonographic characteristics worsen over time would result in better outcomes.

\section{Competing interests}

The authors declare that they have no competing interests.

\section{Authors' contributions}

SM performed the literature review and drafted the manuscript. DM provided the radiology images and participated in drafting/revising the manuscript. LP provided the pathology images and participated in drafting/ revising the manuscript. SW helped to provide the surgery perspective and edited the manuscript. JZ assisted with the literature review and drafting of the manuscript. All authors read and approved the final manuscript.

\section{Acknowledgements}

The authors would like to acknowledge Jeffrey R. Garber, M.D. for helpful discussions. We also acknowledge the support of the Diana and Richard C. Strauss Professorship in Biomedical Research and the Mr. and Mrs. Bruce G. Brookshire Professorship in Medicine at UTSW Medical Center.

\section{Author details}

'Department of Internal Medicine, Division of Endocrinology and Metabolism, The University of Texas Southwestern Medical Center, Dallas, Texas 75390, USA. ${ }^{2}$ Department of Radiology, Neurology and Neurotherapeutics, The University of Texas Southwestern Medical Center Dallas, TX 75390, USA. ${ }^{3}$ Department of Pathology, The University of Texas Southwestern Medical Center, Dallas, TX, USA75390. ${ }^{4}$ Department of Surgery, The University of Texas Southwestern Medical Center, Dallas, TX 75390, USA.

Received: 20 March 2013 Accepted: 19 April 2013

Published: 4 May 2013

\section{References}

1. Vander JB, Gaston EA, Dawber TR: The significance of nontoxic thyroid nodules. Final report of a 15-year study of the incidence of thyroid malignancy. Ann Intern Med 1968, 69:537-540.
2. Reiners C, Wegscheider K, Schicha H, Theissen P, Vaupel R, Wrbitzky R, Schumm-Draeger PM: Prevalence of thyroid disorders in the working population of Germany: ultrasonography screening in 96,278 unselected employees. Thyroid 2004, 14:926-932.

3. Guth S, Theune U, Aberle J, Galach A, Bamberger CM: Very high prevalence of thyroid nodules detected by high frequency $(13 \mathrm{MHz})$ ultrasound examination. Eur J Clin Invest 2009, 39:699-706.

4. Mortensen JD, Woolner LB, Bennett WA: Gross and microscopic findings in clinically normal thyroid glands. J Clin Endocrinol Metab 1955, 15:1270-1280

5. Hegedus L: Clinical practice. The thyroid nodule. N Engl J Med 2004, 351:1764-1771.

6. Cooper DS, Doherty GM, Haugen BR, Kloos RT, Lee SL, Mandel SJ, Mazzaferri EL, Mclver B, Pacini F, Schlumberger M, et al: Revised American Thyroid Association management guidelines for patients with thyroid nodules and differentiated thyroid cancer. Thyroid 2009, 19:1167-1214.

7. Garber JR: Thyroid nodules 2006: managing what has been known for over 50 years. Hormones (Athens) 2006, 5:179-186.

8. Bahn RS, Burch HB, Cooper DS, Garber JR, Greenlee MC, Klein I, Laurberg P, McDougall IR, Montori VM, Rivkees SA, et al: Hyperthyroidism and other causes of thyrotoxicosis: management guidelines of the American Thyroid Association and American Association of Clinical Endocrinologists. Endocr Pract 2011, 17:456-520.

9. Senyurek Giles Y, Tunca F, Boztepe H, Kapran Y, Terzioglu T, Tezelman S: The risk factors for malignancy in surgically treated patients for Graves' disease, toxic multinodular goiter, and toxic adenoma. Surgery 2008, 144:1028-1036. discussion 1036-1027.

10. Cakir M, Arici C, Alakus H, Altunbas H, Balci MK, Karayalcin U: Incidental thyroid carcinoma in thyrotoxic patients treated by surgery. Horm Res 2007, 67:96-99.

11. Cappelli C, Braga M, De Martino E, Castellano M, Gandossi E, Agosti B, Cumetti D, Pirola I, Mattanza C, Cherubini L, Rosei EA: Outcome of patients surgically treated for various forms of hyperthyroidism with differentiated thyroid cancer: experience at an endocrine center in Italy. Surg Today 2006, 36:125-130.

12. Foppiani L, Del Monte P, Marugo A, Arlandini A, Sartini G, Marugo M, Bernasconi D: Heterogeneous malignancy in toxic thyroid nodules. J Endocrinol Invest 2005, 28:294-295.

13. Sahin M, Guvener ND, Ozer F, Sengul A, Ertugrul D, Tutuncu NB: Thyroid cancer in hyperthyroidism: incidence rates and value of ultrasoundguided fine-needle aspiration biopsy in this patient group. J Endocrinol Invest 2005, 28:815-818.

14. Gabriele R, Letizia C, Borghese M, De Toma G, Celi M, Izzo L, Cavallaro A: Thyroid cancer in patients with hyperthyroidism. Horm Res 2003, 60:79-83.

15. Harach HR, Sanchez SS, Williams ED: Pathology of the autonomously functioning (hot) thyroid nodule. Ann Diagn Pathol 2002, 6:10-19.

16. Vaiana R, Cappelli C, Perini P, Pinelli D, Camoni G, Farfaglia R, Balzano R, Braga M: Hyperthyroidism and concurrent thyroid cancer. Tumori 1999, 85:247-252

17. Zanella E, Rulli F, Muzi M, Sianesi M, Danese D, Sciacchitano S, Pontecorvi A: Prevalence of thyroid cancer in hyperthyroid patients treated by surgery. World J Surg 1998, 22:473-477. discussion 477-478.

18. Terzioglu T, Tezelman S, Onaran Y, Tanakol R: Concurrent Hyperthyroidism and Thyroid-Carcinoma. Brit J Surg 1993, 80:1301-1302.

19. Pacini F, Elisei R, Di Coscio GC, Anelli S, Macchia E, Concetti R, Miccoli P, Arganini M, Pinchera A: Thyroid carcinoma in thyrotoxic patients treated by surgery. J Endocrinol Invest 1988, 11:107-112.

20. Smith M, McHenry C, Jarosz H, Lawrence AM, Paloyan E: Carcinoma of the thyroid in patients with autonomous nodules. Am Surg 1988, 54:448-449.

21. Hamburger $\mathrm{J}$ : Solitary autonomously functioning thyroid lesions. Diagnosis, clinical features and pathogenetic considerations. Am J Med 1975, 58:740-748.

22. Horst W, Rosler H, Schneider C, Labhart A: 306 cases of toxic adenoma: clinical aspects, findings in radioiodine diagnostics, radiochromatography and histology; results of $131-\mathrm{I}$ and surgical treatment. J Nucl Med 1967, 8:515-528.

23. Karanchi $H$, Hamilton DJ, Robbins RJ: Hurthle cell carcinoma of the thyroid presenting as thyrotoxicosis. Endocr Pract 2012, 18:e5-9.

24. Yalla NM, Reynolds LR: Hurthle cell thyroid carcinoma presenting as a "hot" nodule. Endocr Pract 2011, 17:e68-72. 
25. Bommireddipalli S, Goel S, Gadiraju R, Paniz-MondolFi A, DePuey EG: Follicular variant of papillary thyroid carcinoma presenting as a toxic nodule by I-123 scintigraphy. Clin Nucl Med 2010, 35:770-775.

26. Giovanella L, Fasolini F, Suriano S, Mazzucchelli L: Hyperfunctioning solid/ trabecular follicular carcinoma of the thyroid gland. J Oncol 2010, 2010.

27. Tfayli HM, Teot LA, Indyk JA, Witchel SF: Papillary thyroid carcinoma in an autonomous hyperfunctioning thyroid nodule: case report and review of the literature. Thyroid 2010, 20:1029-1032.

28. Azevedo MF, Casulari LA: Hyperfunctioning thyroid cancer: a five-year follow-up. Ara Bras Endocrinol Metabol 2010, 54:78-80.

29. Uludag M, Yetkin G, Citgez B, Isgor A, Basak T: Autonomously functioning thyroid nodule treated with radioactive iodine and later diagnosed as papillary thyroid cancer. Hormones (Athens) 2008, 7:175-179.

30. Nishida AT, Hirano S, Asato R, Tanaka S, Kitani Y, Honda N, Fujiki N, Miyata K, Fukushima $\mathrm{H}$, Ito J: Multifocal hyperfunctioning thyroid carcinoma without metastases. Auris Nasus Larynx 2008, 35:432-436.

31. Kim TS, Asato R, Akamizu T, Harada D, Nakashima Y, Higashi T, Yamamoto $\mathrm{N}$, Tamura $\mathrm{Y}$, Tamaki $\mathrm{H}$, Hirano S, et al: A rare case of hyperfunctioning papillary carcinoma of the thyroid gland. Acta Otolaryngol Supp/ 2007, 127:55-57.

32. Niepomniszcze H, Suarez H, Pitoia F, Pignatta A, Danilowicz K, Manavela M, Elsner B, Bruno OD: Follicular carcinoma presenting as autonomous functioning thyroid nodule and containing an activating mutation of the TSH receptor (T620l) and a mutation of the Ki-RAS (G12C) genes. Thyroid 2006, 16:497-503.

33. Bitterman A, Uri O, Levanon A, Baron E, Lefel O, Cohen O: Thyroid carcinoma presenting as a hot nodule. Otolaryngol Head Neck Surg 2006, 134:888-889.

34. Majima T, Doi K, Komatsu Y, Itoh H, Fukao A, Shigemoto M, Takagi C, Corners J, Mizuta N, Kato R, Nakao K: Papillary thyroid carcinoma without metastases manifesting as an autonomously functioning thyroid nodule. Endocr J 2005, 52:309-316.

35. Gozu H, Avsar M, Bircan R, Sahin S, Ahiskanali R, Gulluoglu B, Deyneli O, Ones T, Narin Y, Akalin S, Cirakoglu B: Does a Leu 512 Arg thyrotropin receptor mutation cause an autonomously functioning papillary carcinoma? Thyroid 2004, 14:975-980.

36. Wong CP, AuYong TK, Tong CM: Thyrotoxicosis: a rare presenting symptom of Hurthle cell carcinoma of the thyroid. Clin Nucl Med 2003 28:803-806.

37. Yaturu S, Fowler MR: Differentiated thyroid carcinoma with functional autonomy. Endocr Pract 2002, 8:36-39.

38. Logani S, Osei SY, LiVolsi VA, Baloch ZW: Fine-needle aspiration of follicular variant of papillary carcinoma in a hyperfunctioning thyroid nodule. Diagn Cytopathol 2001, 25:80-81.

39. Mircescu H, Parma J, Huot C, Deal C, Oligny LL, Vassart G, Van Vliet G: Hyperfunctioning malignant thyroid nodule in an 11-year-old girl: pathologic and molecular studies. J Pediatr 2000, 137:585-587.

40. Camacho P, Gordon D, Chiefari E, Yong S, DeJong S, Pitale S, Russo D, Filetti S: A Phe 486 thyrotropin receptor mutation in an autonomously functioning follicular carcinoma that was causing hyperthyroidism. Thyroid 2000, 10:1009-1012.

41. Bourasseau I, Savagner F, Rodien P, Duquenne M, Reynier P, Guyetant S, Bigorgne JC, Malthiery $Y$, Rohmer V: No evidence of thyrotropin receptor and $\mathrm{G}$ (s alpha) gene mutation in high iodine uptake thyroid carcinoma. Thyroid 2000, 10:761-765.

42. Russo D, Wong MG, Costante G, Chiefari E, Treseler PA, Arturi F, Filetti S, Clark $\mathrm{OH}$ : A Val 677 activating mutation of the thyrotropin receptor in a Hurthle cell thyroid carcinoma associated with thyrotoxicosis. Thyroid 1999, 9:13-17.

43. Cirillo RL Jr, Pozderac RV, Caniano DA, Falko JM: Metastatic pure papillary thyroid carcinoma presenting as a toxic hot nodule. Clin Nucl Med 1998, 23:345-349.

44. Russo D, Tumino S, Arturi F, Vigneri P, Grasso G, Pontecorvi A, Filetti $S$, Belfiore A: Detection of an activating mutation of the thyrotropin receptor in a case of an autonomously hyperfunctioning thyroid insular carcinoma. J Clin Endocrinol Metab 1997, 82:735-738.

45. Siddiqui AR, Karanauskas S: Hurthle cell carcinoma in an autonomous thyroid nodule in an adolescent. Pediatr Radiol 1995, 25:568-569.

46. Mizukami Y, Michigishi T, Nonomura A, Yokoyama K, Noguchi M, Hashimoto T, Nakamura S, Ishizaki T: Autonomously functioning (hot) nodule of the thyroid gland. A clinical and histopathologic study of 17 cases. Am J Clin Pathol 1994, 101:29-35.

47. De Rosa G, Testa A, Maurizi M, Satta MA, Aimoni C, Artuso A, Silvestri E, Rufini $V$, Troncone L: Thyroid carcinoma mimicking a toxic adenoma. Eur J Nucl Med 1990, 17:179-184.

48. Ikekubo K, Hino M, Ito H, Otani M, Yamaguchi H, Saiki Y, Ui K, Habuchi Y, Ishihara T, Mori T: Thyroid carcinoma in solitary hot thyroid lesions on Tc-99m sodium pertechnetate scans. Ann Nucl Med 1989, 3:31-36.

49. Sandler MP, Fellmeth B, Salhany KE, Patton JA: Thyroid carcinoma masquerading as a solitary benign hyperfunctioning nodule. Clin NuC Med 1988, 13:410-415.

50. Nagai GR, Pitts WC, Basso L, Cisco JA, McDougall IR: Scintigraphic hot nodules and thyroid carcinoma. Clin NuCl Med 1987, 12:123-127.

51. Fukata S, Tamai H, Matsubayashi S, Nagai K, Hirota Y, Matsuzuka F, Katayama S, Kuma K, Nagataki S: Thyroid carcinoma and hot nodule. Eur J Nucl Med 1987, 13:313-314.

52. Sobel RJ, Liel Y, Goldstein J: Papillary carcinoma and the solitary autonomously functioning nodule of the thyroid. Isr J Med Sci 1985, 21:878-882.

53. Hoving J, Piers DA, Vermey A, Oosterhuis JW: Carcinoma in hyperfunctioning thyroid nodule in recurrent hyperthyroidism. Eur J NuCl Med 1981, 6:131-132.

54. Khan O, Ell PJ, Maclennan KA, Kurtz A, Williams ES: Thyroid carcinoma in an autonomously hyperfunctioning thyroid nodule. Postgrad Med J 1981, 57:172-175.

55. Hopwood NJ, Carroll RG, Kenny FM, Foley TP Jr: Functioning thyroid masses in childhood and adolescence. Clinical, surgical, and pathologic correlations. J Pediatr 1976, 89:710-718.

56. Sussman L, Librik L, Clayton GW: Hyperthyroidism attributable to a hyperfunctioning thyroid carcinoma. J Pediatr 1968, 72:208-213.

57. Dische S: The radioisotope scan applied to the detection of carcinoma in thyroid swellings. Cancer 1964, 17:473-479.

58. Als C, Gedeon P, Rosler H, Minder C, Netzer P, Laissue JA: Survival analysis of 19 patients with toxic thyroid carcinoma. J Clin Endocrinol Metab 2002, 87:4122-4127

59. Lin JD, Huang MJ, Chao TC, Weng HF, Hsueh C: Prevalence of thyroid cancer in 1894 patients with surgically treated thyroid nodules. Cancer $J$ 1997, 10:217-221.

60. Taneri F, Kurukahvecioglu O, Ege B, Yilmaz U, Tekin EH, Cifter C, Onuk E: Clinical presentation and treatment of hyperthyroidism associated with thyroid cancer. Endocr Regul 2005, 39:91-96.

61. Low SC, Sinha AK, Sundram FX: Detection of thyroid malignancy in a hot nodule by fluorine-18-fluorodeoxyglucose positron emission tomography. Singapore Med J 2005, 46:304-307.

62. Schneider PW, Meier DA, Balon H: A clear cell variant of follicular carcinoma presenting as an autonomously functioning thyroid nodule. Thyroid 2000, 10:269-273.

63. Caplan RH, Abellera RM, Kisken WA: Hurthle cell neoplasms of the thyroid gland: reassessment of functional capacity. Thyroid 1994, 4:243-248.

64. Michigishi T, Mizukami Y, Shuke N, Satake R, Noguchi M, Aburano T, Tonami N, Hisada K: An autonomously functioning thyroid carcinoma associated with euthyroid Graves' disease. J Nucl Med 1992, 33:2024-2026.

65. Rubenfeld S, Wheeler TM: Thyroid cancer presenting as a hot thyroid nodule: report of a case and review of the literature. Thyroidology 1988:63-68.

66. Abdel-Razzak M, Christie JH: Thyroid carcinoma in an autonomously functioning nodule. J Nucl Med 1979, 20:1001-1002.

67. Scott MD, Crawford JD: Solitary thyroid nodules in childhood: is the incidence of thyroid carcinoma declining? Pediatrics 1976, 58:521-525.

68. Fujimoto Y, Oka A, Nagataki S: Occurrence of papillary carcinoma in hyperfunctioning thyroid nodule. Report of a case. Endocrinol Jpn 1972, 19:371-374.

69. Becker FO, Economou PG, Schwartz TB: The occurrence of carcinoma in "hot" thyroid nodules. Report of two cases. Ann Intern Med 1963, 58:877-882

70. Molnar GD, Childs DS Jr, Woolner LB: Histologic evidence of malignancy in a thyroid gland bearing a hot nodule. J Clin Endocrinol Metab 1958, 18:1132-1134

71. Bransom CJ, Talbot CH, Henry L, Elemenoglou J: Solitary toxic adenoma of the thyroid gland. Br J Surg 1979, 66:592-595. 
72. Blum M, Shenkman L, Hollander CS: The autonomous nodule of the thyroid: correlation of patient age, nodule size and functional status. Am J Med Sci 1975, 269:43-50.

73. Burch HB, Shakir F, Fitzsimmons TR, Jaques DP, Shriver CD: Diagnosis and management of the autonomously functioning thyroid nodule: the Walter Reed Army Medical Center experience, 1975-1996. Thyroid 1998, 8:871-880.

74. Iwata M, Kasagi K, Hatabu H, Misaki T, lida Y, Fujita T, Konishi J: Causes of appearance of scintigraphic hot areas on thyroid scintigraphy analyzed with clinical features and comparative ultrasonographic findings. Ann Nucl Med 2002, 16:279-287.

75. Landgarten S, Spencer RP: A study of the natural history of "hot" thyroid nodules. Yale J Biol Med 1973, 46:259-263.

76. Linos DA, Karakitsos D, Papademetriou J: Should the primary treatment of hyperthyroidism be surgical? Eur J Surg 1997, 163:651-657.

77. Hamburger Jl: Evolution of toxicity in solitary nontoxic autonomously functioning thyroid nodules. J Clin Endocrinol Metab 1980, 50:1089-1093.

78. Langer M, Madeddu G, Costanza C: Free thyroxine (FT4) and free triiodothyronine (FT3) in autonomous thyroid nodules. Clin Endocrinol (Oxf) 1979, 11:461-464

79. Alexander EK, Hurwitz S, Heering JP, Benson CB, Frates MC, Doubilet PM, Cibas ES, Larsen PR, Marqusee E: Natural history of benign solid and cystic thyroid nodules. Ann Intern Med 2003, 138:315-318.

80. Hundahl SA, Fleming ID, Fremgen AM, Menck HR: A National Cancer Data Base report on 53,856 cases of thyroid carcinoma treated in the U.S., 1985-1995 [see commetns]. Cancer 1998, 83:2638-2648

81. Pazaitou-Panayiotou K, Michalakis K, Paschke R: Thyroid cancer in patients with hyperthyroidism. Horm Metab Res 2012, 44:255-262.

82. Croom RD 3rd, Thomas CG Jr, Reddick RL, Tawil MT: Autonomously functioning thyroid nodules in childhood and adolescence. Surgery 1987, 102:1101-1108

83. Niedziela M, Breborowicz D, Trejster E, Korman E: Hot nodules in children and adolescents in western Poland from 1996 to 2000: clinical analysis of 31 patients. J Pediatr Endocrinol Metab 2002, 15:823-830.

84. Pacini F: Thyroid microcarcinoma. Best Pract Res Clin Endocrinol Metab 2012, 26:421-429.

85. Sundaraiya S, Dizdarevic S, Miles K, Quin J, Williams A, Wheatley T, Zammitt C Unusual initial manifestation of metastatic follicular carcinoma of the thyroid with thyrotoxicosis diagnosed by technetium Tc $99 \mathrm{~m}$ pertechnetate scan: case report and review of literature. Endocr Pract 2009, 15:458-462.

86. Kim JH, Park JH, Kim SY, Bae HY: Symptomatic calcification of a thyroid lobe and surrounding tissue after radioactive iodine treatment to ablate the lobe. Thyroid 2011, 21:203-205.

doi:10.1186/1756-6614-6-7

Cite this article as: Mirfakhraee et al:: A solitary hyperfunctioning thyroid nodule harboring thyroid carcinoma: review of the literature. Thyroid Research 2013 6:7.

\section{Submit your next manuscript to BioMed Central and take full advantage of:}

- Convenient online submission

- Thorough peer review

- No space constraints or color figure charges

- Immediate publication on acceptance

- Inclusion in PubMed, CAS, Scopus and Google Scholar

- Research which is freely available for redistribution

Submit your manuscript at www.biomedcentral.com/submit
Ciomed Central 